\title{
Study Of Vertical Emulsifier Fault Diagnosis System Based On EMD-Sample Entropy And BP Neural Network
}

\author{
Yue-sheng Wang ${ }^{1,}$,, Yao-wen Sun ${ }^{2, b}$ \\ ${ }^{1}$ Institute of Automation, Hangzhou Dianzi University, Hangzhou 310018, China; \\ ${ }^{2}$ Institute of Automation, Hangzhou Dianzi University, Hangzhou 310018, China. \\ Aijie009@126.com, baccountmanage@126.com,
}

Keywords: Emulsifier, Sample Entropy, EMD, BP Neural Network.

\begin{abstract}
Aiming at the nonlinear characteristics of the fault vibration signal of the emulsifier, a feature extraction method based on Empirical Mode Decomposition (EMD) and Sample Entropy (SampEn) is proposed.In this method, the original vibration signal is decomposed into a finite number of intrinsic mode functions by EMD, then we select the Sample Entropy (SampEn) of the intrinsic mode function which contains the main failure information as the characteristic parameter, and BP neural network is used to diagnose the faults of the emulsifier in this study. Experiments verified that the BP neural network diagnosis method can get better fault diagnosis effect by using the EMD pretreatment to extract the Sample Entropy as the characteristic parameter.
\end{abstract}

\section{Introduction}

Emulsifier is the key equipment in the production of emulsion explosive [1,2]. Multiple faults in the practical operation of the emulsifier, such as rotor scratch, bearing fault, loosening, fracture, rotating stall, will result in the dynamic signal non stationarity appeared. Nonlinear feature extraction method has attracted wide attention in recent years. The concept of approximate entropy is proposed from the viewpoint of measuring the complexity of nonlinear time series in Pincus [3]. Sample entropy (SampEn) is proposed by Richman based on approximate entropy [4]. Sample entropy does not depend on the length of time series, and has good anti disturbance ability, which overcomes the defects in the approximate entropy calculation.

Because of the complex mechanism of the emulsifier, the characteristic signal is not obvious, it is very difficult to extract the fault information effectively by using the conventional signal processing method. Empirical Mode Decomposition (EMD) is a new method to deal with nonlinear and non-stationary signals, which is proposed by Huang et al [5]. The essence of the method is that the complex signal is decomposed into a finite number of intrinsic mode functions (IMF).

BP Neural Network is a kind of multi-layer feed forward network which is trained by error back propagation algorithm [6]. The advantage is that the network can approach any nonlinear function and has good generalization ability as long as there is enough hidden layer and hidden node. BP Neural Network is one of the most widely used and successful artificial Neural Networks in the field of fault diagnosis.

\section{Sample Entropy}

For a known $N$ data points from a time series $\{x(i) \mid 1 \leq i \leq N\}$, Sample Entropy can be defined as follows [7]:

(1)Given dimension $m$, construct a set of $m$-dimensional vector $X(i)$ defined by

$$
X(i)=[x(i), x(i+1), \ldots, x(i+m-1)], i=1, \ldots, N-m+1
$$

(2)Define the distance between vectors $X(i)$ and $X(j), d[X(i), X(j)]$ as the absolute maximum difference between their scalar components:

$$
d[X(i), X(j)]=\max _{k=0,1, \ldots, m-1}(|x(i+k)-x(j+k)|)
$$


(3)For a given $X(i)$, count the number of $j(1 \leq j \leq N-m, j \neq i)$, denoted as $B_{i}$, such that the distance between $X(i)$ and $X(j)$ is less than or equal to $r$. Then, for $1 \leq i \leq N-m$ :

$$
B_{i}^{m}(r)=\frac{1}{N-m-1} B_{i}
$$

(4)Define $B^{m}(r)$ as

$$
B^{m}(r)=\frac{1}{N-m} \sum_{i=1}^{N-m} B_{i}^{m}(r)
$$

(5)Increase the dimension to $m+1$ and calculate $B^{m+1}(r)$,

$$
B^{m+1}(r)=\frac{1}{N-m} \sum_{i=1}^{N-m} B_{i}^{m+1}(r)
$$

Thus, $B^{m}(r)$ is the probability that two sequences will match for $m$ points, whereas $B^{m+1}(r)$ is the probability that two sequences will match for $m+1$ points. Finally, Sample Entropy can be defined as

$$
\operatorname{SampEn}(m, r)=\lim _{N \rightarrow \infty}\left\{-\ln \frac{B^{m+1}(r)}{B^{m}(r)}\right\}
$$

which is estimated by the statistic

$$
\operatorname{SampEn}(m, r)=-\ln \frac{B^{m+1}(r)}{B^{m}(r)}
$$

Although $m$ and $r$ are critical in determining the outcome of SampEn, no guidelines exist for optimizing their values. The most widely established values for $m$ and $r$ are, $m=1$ or $m=2$ and $r$ between 0.1 and 0.25 times the standard deviation of the original time series. In this study, determines parameters $m=1, r=0.2, N=500$.

However, under the high-speed operation of the emulsifier, and complex environment interference, if more data in the presence of mechanical failure, different fault sample entropy similarity is high, resulting in indistinguishable, it can not reach the purpose of fault classification. Table.1 lists the SampEn value of the radial and axial vibrations of the emulsifier at different faults.

Table 1 SampEn value of different emulsifier fault types

\begin{tabular}{ccccc}
\hline \hline SampEn value & $\begin{array}{c}\text { Bearing outer ring } \\
\text { crack }\end{array}$ & $\begin{array}{c}\text { Bearing rolling } \\
\text { element defect }\end{array}$ & $\begin{array}{c}\text { Rotor } \\
\text { friction }\end{array}$ & $\begin{array}{c}\text { Rotor } \\
\text { misalignment }\end{array}$ \\
\hline $\begin{array}{c}\text { Radial } \\
\text { vibration }\end{array}$ & 1.415 & 1.802 & 1.542 & 2.185 \\
\hline Axial vibration & 0.856 & 0.902 & 0.714 & 1.081 \\
\hline \hline
\end{tabular}

From the table can be seen that either radial or axial SampEn values caused by crack of bearing outer ring and rotor friction fault are quite similar, So it is limited to use the sample entropy of the original signal to diagnose the fault of the emulsifier. The main reason is that the information provided by the SampEn of the original signal is very limited, and the deep information contained in the signal can not be reflected. If we select a good signal decomposition method, decompose original signal into a number of components, taking the sample entropy of each component as the fault feature, we can overcome these limitations, and better classify the fault.

\section{Empirical Mode Decomposition(EMD)}

The empirical mode decomposition method proposed is designed to decompose the fluctuations or trends of different scales in the signal step by step to generate a series of IMF components of the characteristic time scale, and the EMD algorithm is as follows [8]: 


$$
x(t)=\sum_{i=1}^{n} c_{i}(t)+r_{n}(t)
$$

$x(t)$ for the original vibration signal; $c_{i}(t)$ for each decomposition of the IMF components; $r_{n}(t)$ for the residual function which reflects the overall trend of the signal on behalf of the signal.

EMD decomposition is carried out on the radial vibration signal of the emulsifier fault caused by the outer ring crack and rotor friction respectively, and the results are shown in Figure 1.Two kinds of fault vibration signals are decomposed into $8 \mathrm{IMF}$ components and 1 residual component, each IMF component contains a different time characteristic scale, which makes the fault information exposed at different characteristic scales.
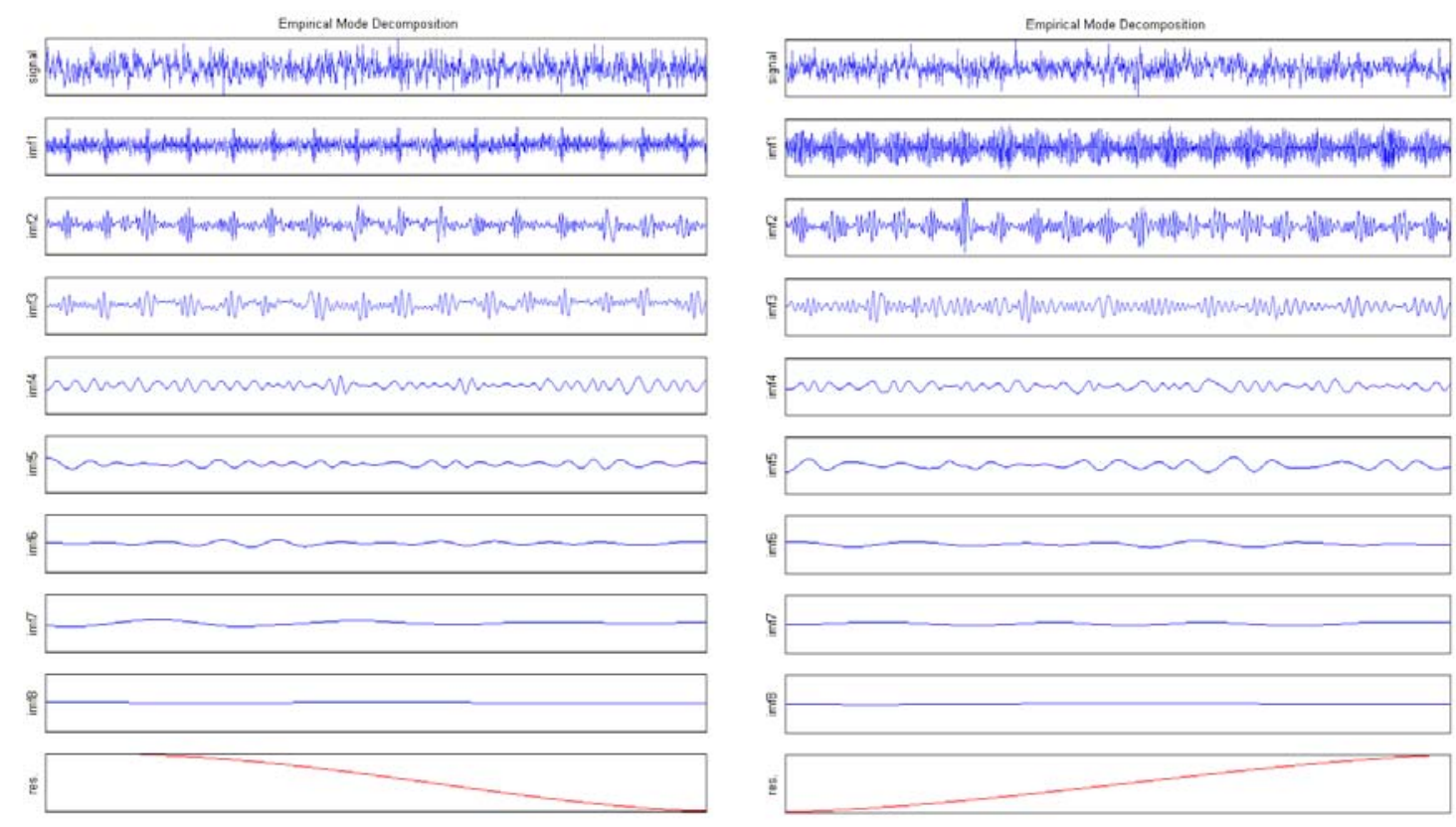

Fig.1 EMD decomposition map of outer ring crack (Left) and rotor friction (Right)

The frequency of the IMF 1 8 component is from high to low, and the mechanical fault information appears in the high speed operation of the emulsifier is concentrated in the high frequency section. The first 3 IMF components were selected, and calculate SampEn of 3 IMF components respectively shown in Table 2.The SampEn of the 3 IMF components is used as the element, radial feature vector can be defined as

$$
\mathbf{E}_{\mathbf{R}}=\left[\operatorname{SampEn}_{1}, \ldots ., \operatorname{SampEn}_{4}\right]^{\mathbf{T}}
$$

Both axial and radial vibration of the emulsifier are monitored at the same time in system. We can define a two-dimensional feature vector group $\mathbf{T}=\left[\mathbf{E}_{\mathbf{A}}, \mathbf{E}_{\mathbf{R}}\right]$, which is composed of axial feature $\mathbf{E}_{\mathbf{A}}$ and radial feature $\mathbf{E}_{\mathbf{R}}$, set as the fault feature.

Table 2 SampEn value of outer ring crack and rotor friction fault (radial vibration)

\begin{tabular}{cccc}
\hline \hline component & IMF 1 & IMF 2 & IMF 3 \\
\hline outer ring crack & 2.142 & 0.962 & 0.208 \\
\hline rotor friction & 1.758 & 1.210 & 0.648 \\
\hline \hline
\end{tabular}

\section{BP Neural Network Emulsifier Fault Diagnosis}

In the field of fault diagnosis, the most effective is the forward Neural Network [9]. Since the network in the learning process using the Back-Propagation algorithm, so the neural network also known as the BP Neural Network.

The fault diagnosis model of emulsifier is shown in Figure 2. The diagnosis process is as follows: 
(1)The signal is collected by the acceleration sensor, and then decomposed by EMD, so that axial IMF components and radial IMF components are obtained;

(2)Take each of the first 3 IMF components, calculate SampEn of each IMF component, construct a two-dimensional feature group $\mathbf{T}=\left[\mathbf{E}_{\mathbf{A}}, \mathbf{E}_{\mathbf{R}}\right], \mathbf{E}_{\mathbf{A}}$ is the axial SampEn feature vector, $\mathbf{E}_{R}$ is the radial SampEn feature vector;

(3)The training sample data are used to train the BP Neural Network to adjust the threshold parameters;

(4)Sample data is input to the BP Neural Network, calculate the output vector, determine the fault type and output alarm.

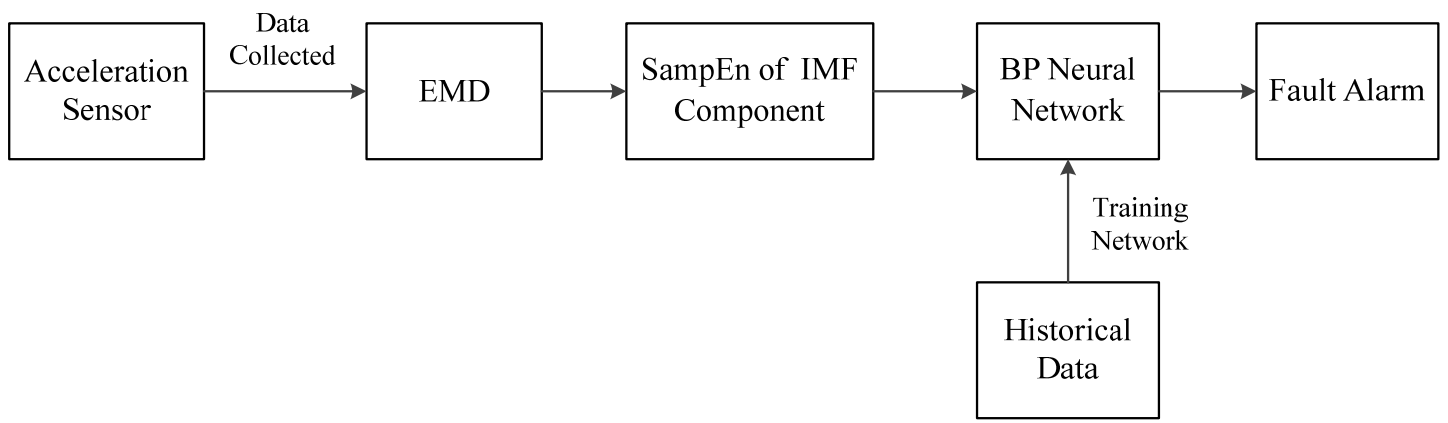

Fig.2 BP Network fault diagnosis system for emulsifier

\section{Experiment Results}

The common faults of the emulsifier are bearing outer ring crack, bearing rolling element defect and rotor friction. When there is a certain type of fault in the emulsifier, the expected output of the corresponding fault elements in the network output vector is 1 , and other elements of the expected output are 0 . When the failure probability is greater than 0.8 , the fault type can be basically determined. The matrix codes for output fault are, bearing outer ring crack $[0,0,1]$, bearing rolling element defect $[0,1,0]$, rotor friction $[1,0,0]$, respectively. Collect vibration data 100 groups, randomly selected 80 groups for the sample training, and the remaining 20 groups for diagnostic testing. The fault test is done with the EMD-SampEn, and compared with the fault test based on the SampEn of the original signal, the results are shown in Table 3.Although the two methods can identify the mechanical faults of the emulsifier, the network method based on the EMD-SampEn to extract the characteristic parameters has higher network identification ability.

Table 3 Fault diagnosis test results

\begin{tabular}{cccc}
\hline \hline Type & $\begin{array}{c}\text { Bearing outer ring } \\
\text { crack [0,0,1] }\end{array}$ & $\begin{array}{c}\text { Bearing rolling } \\
\text { element defect }[0,1,0]\end{array}$ & Rotor friction [1,0,0] \\
\hline $\mathbf{T}_{1}$ & $(0.762,2.184)$ & $(1.141,2.854)$ & $(0.525,1.841)$ \\
\hline $\mathbf{T}_{2}$ & $(0.515,0.911)$ & $(0.874,2.314)$ & $(0.210,1.147)$ \\
\hline $\mathbf{T}_{3}$ & $(0.167,0.189)$ & $(0.441,1.406)$ & $(0.042,0.655)$ \\
\hline $\begin{array}{c}\text { Network } \\
\text { output }\end{array}$ & $(0.0175,0.0635,0.9414)$ & $(0.0195,0.8054,0.1417)$ & $(0.8649,0.0502,0.0187)$ \\
\hline $\begin{array}{c}\text { Network } \\
\text { output }\end{array}$ & $(0.2081,0.1589,0.7445)$ & $(0.4241,0.7106,0.0153)$ & $(0.8241,0.2106,0.3153)$ \\
\hline \hline
\end{tabular}

\section{Summary}

In this paper, a feature extraction method based on EMD-SampEn is proposed to diagnose failure combined with BP Neural Network. Tests show that the fault diagnosis system established by this method can quickly and accurately find out the location of the fault and the cause of the failure, so it 
can be effectively applied to the actual production.In addition, it provides a new way to solve the problem of fault diagnosis in other fields.

\section{References}

[1] Wang Xu-guang, Emulsion Explosive (Second Edition), Beijing, Metallurgical Industry Press, 2008.

[2] Wu Long-xiang, Pei Hai-xing et al., The safety research on continue production process of the Emulsion Explosive[J], Explosive Equipment, 2003, 32(2).

[3]Pincus S M, Approximate entropy as a measure of system complexity[C]. Proc. Natl. Acad. Sci. USA, 1991, 88: 2297-2301.

[4]Richman J S Moorman J R. Physiological time-series analysis using approximate entropy and sample entropy[J]. American Journal of Physiology-Heart and Circulatory Physiology, 2000, 278(6): H2039-H2049.

[5]Huang N E, Shen Z, Long S R, et al. The empirical mode decomposition and the Hibert Spectrum for nonlinear and non-stationary time series analysis[C]. Proc. R. Soc. Lond. A, 1998, 454: 903-995.

[6]Xu Bing-zheng. Neural Network Theory And Application. Guangzhou: South China University of Technology Press, 1994.

[7]Liu Hui, He Wei-xing, Chen Xiao-ping. Comparison between approximate entropy and sample entropy method for biological time series[J]. Journal of instrument and meter, 2004, 25(zl): 806-807, 812.

[8]Cao Chong-feng. Research on mechanical fault diagnosis and vibration method based on EMD[D]. Hangzhou: Doctoral Dissertation of Zhejiang University, 2009: 20-28.

[9]Yan Yan-hu. Zhong Bing-lin, Huang Ren. Neural network technology and its application in fault diagnosis of rotating machinery[J]. Journal of Vibration Engineering, 1993. 